\title{
EVALUATION OF STATIC AND DYNAMIC CHARACTERISTICS OF HYDRODYNAMIC SPIRAL-GROOVED JOURNAL BEARINGS USING THE CFD
}

\author{
Janardhan Chander Matlapudi - Prudhvi Kanth Gunti - Datta Sriram Sigatapu - Niranjana Behera*
}

School of Mechanical Engineering, VIT University, Vellore, Tamilnadu-632014, India

\begin{tabular}{l}
\hline ARTICLE INFO \\
\hline Article history: \\
Received: 3.6 .2017 . \\
Received in revised form: 22.10 .2017$. \\
Accepted: 7.12 .2017$. \\
\hline Keywords: \\
spiral-groove; \\
load carrying capacity; \\
stiffness coefficient; \\
damping coefficient; \\
CFD \\
\hline
\end{tabular}

DOI: http://doi.org/10.30765/er.39.2.9

\begin{abstract}
:
Performance of plain journal bearings can be different by inserting grooves of different crosssections either on the journal or a bearing surface. This paper presents the prediction of load carrying capacity, stiffness, and damping coefficients of spiral-grooved bearing using the Computational Fluid dynamics (CFD) technique. The spiral grooves have been provided on the rotor surface. The error between the predicted values of these parameters using the CFD and the experimental value is low. A comparative analysis of performance has also been carried out between a grooved journal bearing and a plain journal bearing. The load carrying capacity of plain journal bearing is high when compared to spiral grooved journal bearing. At higher eccentrcity ratios, stability of a spiral-grooved journal bearing is comparatively better than a plain bearing.
\end{abstract}

the synchronous vibration caused by forces due to unbalance of shaft. The second kind of instability is self-excited non-synchronous vibration (or lowfrequency whirl) caused due to oil film forces occurring at low eccentricities as given in [1].

The performance of journal bearings can be different if either the inside surface of bearings or outer surface of rotor are grooved. The groove may be straight, helical, or spiral. When the journal rotates, the grooves induce a self-pumping action to the lubricant which causes an additional pressure development in the bearing. This additional pressure causes a pre-load on the rotor and also improves the stability of bearing. [2] studied that the load carrying capacity of journal bearings with oil grooves reduces by 30 to 70 percent if the oil grooves are kept at the maximum pressure location. [3] studied that the grooves should not be positioned

\footnotetext{
* Corresponding author.

E-mail address: beheranira@rediffmail.com
} 
in the load carrying area, since it may interfere with the natural hydrodynamic action of the bearing. It should be placed near the maximum film thickness region. [4] studied the performance of two-groove journal bearings using genetic algorithm by keeping grooves at different locations. It has been concluded that the performance of bearing may not be necessarily better if the two grooves are located $180^{\circ}$ apart. [5] used a perturbation technique to calculate the stiffness and damping coefficients for a water-lubricated journal bearing with three equally spaced axial grooves.

For the last few decades, many researchers have used commercial computational fluid dynamics (CFD) programs for investigating the parameters in journal bearings. In these programs, the full NavierStokes equations have been solved. The CFD packages can be applicable to very complex geometries as well. [6] used the CFD approach to model the fluid flow in a journal bearing with a three equi-spaced axial grooves using water as a lubricant. They found that the location of the maximum pressure in the clearance space occurs near to the outlet side instead of at the central plane. [7] developed CFD model uses Fluent software for analyzing the flow parameters involved in a hydrodynamic journal bearing with a Bingham lubricant. They concluded that the load carrying capacity, film pressure, and frictional force for a Bingham lubricant (a non-Newtonian fluid) are more than that for a lubricant of Newtonian fluid. [8] modeled using the CFD approach to study the static and dynamic characteristics of grooved journal bearings with water as lubricant. They observed that eight-grooved bearing induce higher value of stiffness or damping than a three-grooved bearing, hence it can provide higher stability. [9] conducted numerical simulation using the CFD technique in order to study the effect of microgrooves on rotor with a journal bearing. They analyzed that the difference in predicted load carrying capacity between a micro-grooved and a plain rotor in journal bearing is negligible. [10] conducted a comparative analysis between a threelobe journal bearing and a plain journal bearing using the CFD. They found that a lobed bearing can carry a higher load than a plain bearing. [11] implemented the CFD technique for a lobed journal bearing and concluded that at higher operating speeds lobed journal bearing shows more life span than a plain journal bearing. In few researches the CFD has been applied to analyze the performance of grooved bearing. But so far the CFD has not been applied on study of spiral-grooved journal bearing. In the present work, the CFD analysis on a spiral grooved journal bearing with grooves cut on the surface of journal has been carried out. The load carrying capacity, stiffness and damping coefficients have been predicted from the CFD simulation result and also compared with the experimental data.

\section{Model of fluid film in spiral-grooved journal bearing}

Solid works modeling software has been implemented to model the fluid film by considering the dimensions from the experimental setup presented in [12] (Fig. 1). In the setup, the spiral grooves have been cut on the rotor, hence the model of fluid film has spiral grooves cut on its inside surface. The dimensions of the spiral-grooved bearing are presented in Table 1.

\section{Table 1. Dimensions of grooved-journal bearing}

\begin{tabular}{|l|c|}
\hline Specifications & \multicolumn{2}{l}{ Value } \\
\hline Length $\left(\mathrm{l}_{0}\right)$ & $86 \mathrm{~mm}$ \\
\hline Inner diameter $\left(\mathrm{d}_{1}\right)$ & $99.76 \mathrm{~mm}$ \\
\hline Outer diameter $\left(\mathrm{d}_{2}\right)$ & $100 \mathrm{~mm}$ \\
\hline Radial clearance $\left(\mathrm{F}_{\mathrm{r}}\right)$ & $0.012 \mathrm{~mm}$ \\
\hline Width of groove & $7.84 \mathrm{~mm}$ \\
\hline Depth of groove & $0.32 \mathrm{~mm}$ \\
\hline Groove angle & $33^{\circ}$ \\
\hline No. of grooves & 15 \\
\hline
\end{tabular}

The quality of mesh plays an important role in the stability of numerical computation. So checking the quality of mesh is essential. Choosing the mesh type is also important for simulation. ANSYS uses different mesh types mainly hexahedral, tetrahedral, and polyhedral [13]. In the present work, as the spiral grooved geometry is complex, tetrahedral mesh has been used (Fig.2). The quality of mesh has been identified by the magnitude of the aspect ratio. In this work, three meshes with different number of elements (3 lakhs, 13 lakhs and 30 lakhs) have been considered. The aspect ratio was varying from 1 to 92, 1 to 38 and 1 to 22 for 3 lakhs, 13 lakhs and 30 
lakhs respectively. Quality of mesh is better if the aspect ratio is near to 1 . In these meshes, higher number of elements was with the low aspect ratios.

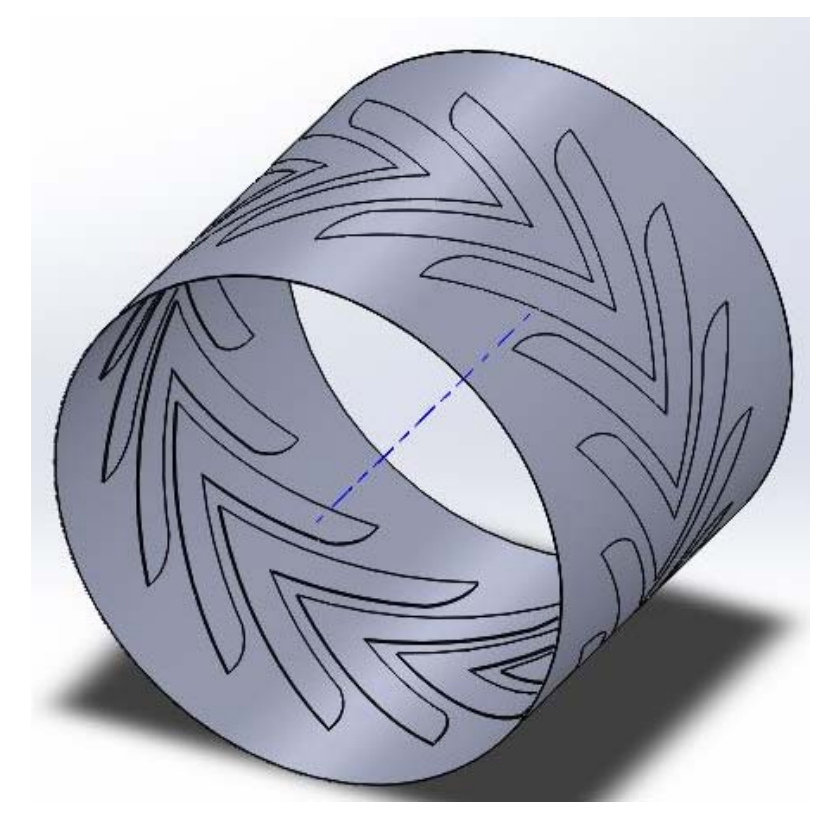

Fig. 1 Model of the fluid film.

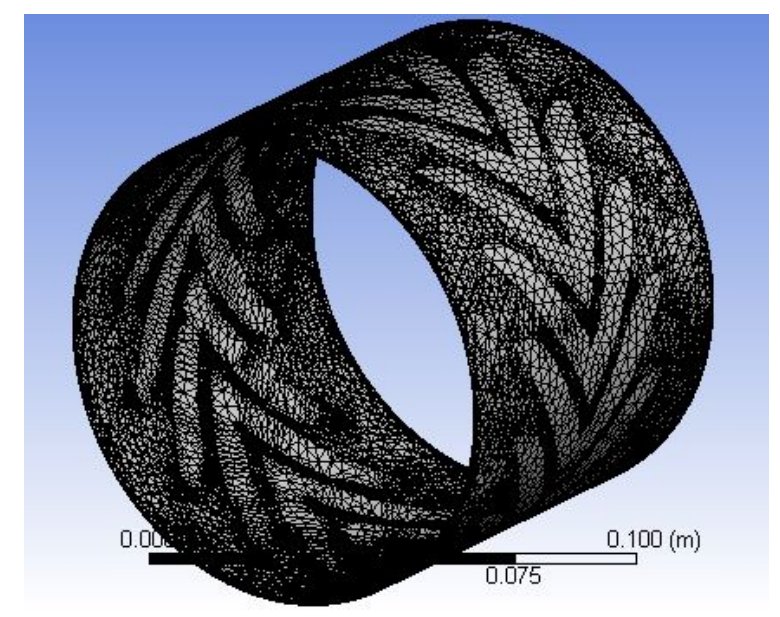

Fig 2: Mesh of the fluid film.

\section{Numerical Simulation}

Computational fluid dynamics uses the equations of Navier-Stokes which are expressed as following. Conservation equation of mass or continuity equation can be written as:

$$
\nabla \cdot(\rho u)=0
$$

Conservation of momentum equation is

$$
\nabla \cdot(\rho u u)=\nabla \cdot \tau-\nabla P
$$

where, the $1^{\text {st }}$ and the $2^{\text {nd }}$ term on right hand side of the equation are shear force and pressure force terms respectively.

For computational fluid dynamics simulation software package FLUENT 15.0 has been used. Paraffin oil has been used as the fluid which has density $870 \mathrm{~kg} / \mathrm{m}^{3}$, viscosity $0.02784 \mathrm{~kg} / \mathrm{m} \cdot \mathrm{s}$. Since the Reynolds number is 0.24 for the operating condition with a rotor speed $350 \mathrm{rpm}$, a viscous laminar model has been considered and the effect of turbulence has been neglected. The inner surface of the fluid film is considered as moving wall since the rotor contacts to the inner surface. Similarly, the outer wall of the film which is in contact with bearing has been considered as stationary wall. Two ends of the bearing are taken as walls assuming that there is no leakage of fluid through the two ends of bearing. Hence a "wall" boundary condition has been chosen at the inlet and outlet. The second order discretization for pressure and the second order upwind for momentum have been used. SIMPLE scheme has been used for pressure-velocity coupling. Simulation was conducted till a converged solution has been obtained.

The pressure forces on each element may be heading in a definite direction. These forces can be resolved in three different directions $\mathrm{x}, \mathrm{y}$ and $\mathrm{z}$ axes. Under steady state the load is vertical. For calculating the load, equivalent y-direction pressure forces are calculated by reducing the other components ( $\mathrm{x}$ and $\mathrm{z}$ ) to zero.

The Mesh Independence test has been performed by successively using smaller cell sizes for the calculations. The test was performed for three different numbers of cells in the increasing manner of 3lakh, 13 lakh and 30 lakh cells [14]. The nondimensional load carrying capacities for different eccentricities have been presented in the following Table 2. By conducting this test, it is found that the load carrying capacity rapidly increased for 3lakh to 13 lakh cells, but did not change much for 13lakh to 30 lakh cells. So 13 lakhs cells mesh has been used for the CFD analysis in this study. For each model the simulation is conducted till a converged solution of load carrying capacity reached. After certain number of iterations, the load carrying capacity was calculated in order to check for convergence. 
Table 2: Mesh independence test

\begin{tabular}{|c|c|c|c|}
\hline \multirow{2}{*}{ Eccentricity } & \multicolumn{3}{|c|}{ Non-dimensional load carrying } \\
& capacity \\
\cline { 2 - 4 } & $\begin{array}{c}\text { 3 lakh } \\
\text { cells }\end{array}$ & $\begin{array}{c}\text { 13 lakh } \\
\text { cells }\end{array}$ & $\begin{array}{c}\text { 30 lakh } \\
\text { cells }\end{array}$ \\
\hline 0.1 & 0.15 & 0.21 & 0.22 \\
\hline 0.2 & 0.32 & 0.43 & 0.43 \\
\hline 0.3 & 0.48 & 0.66 & 0.66 \\
\hline 0.4 & 0.64 & 0.86 & 0.87 \\
\hline 0.5 & 0.88 & 1.21 & 1.21 \\
\hline 0.6 & 1.09 & 1.48 & 1.49 \\
\hline
\end{tabular}

\section{Calculation of static and dynamic parameters}

FLUENT has the option of calculating the static and dynamic pressures. The total pressure is a sum of static pressure and dynamic pressure. Static pressure is the pressure by the fluid when rotor is in static position. Dynamic pressure is based on the kinetic energy of flowing fluid due to motion of rotor.

After a converged solution was obtained, the static pressure, dynamic pressure, and face area (normal to radial and tangential direction) data are extracted. Static pressures have been used to calculate the static load or load carrying capacity. This was possible by calculating the force only in the vertical direction (i.e. y-direction), while considering the forces in other two directions to be zero.

Dynamic characteristics such as stiffness and damping coefficient are necessary to calculate, since they are the analogous properties of spring and damper in simple vibration theory. Dynamic pressures predicted from the CFD simulation can be used to calculate stiffness and damping coefficient. To extract the dynamic pressures, in addition to spinning shaft, it is necessary to impose the whirling of a shaft. For the present case, spinning and whirling of the shaft have been assumed to take place in the same sense of direction. Hence to implement the concept of whirling of the shaft in FLUENT, the bearing surface was rotated opposite to that spinning of the shaft.

In Fig. 3, the positions of the rotor and fluid domain are moving all the time. This problem is transient in stationary frame. For this analysis it would need a moving mesh. But transient analysis with a moving mesh can be avoided by employing coordinate transformation from a transient problem in stationary frame (Fig. 3) into a steady one (Fig. 4).
In Fig. 4, rotating frame of reference is whirling rotor. The rotor spins clockwise with speed $\omega$, the frame rotates clockwise at speed $\Omega$ and the stator spins anticlockwise at a speed $\Omega$. In this manner the problem reduces to a steady state without whirling of the rotor and calculation of all parameters is performed in the rotating frame.

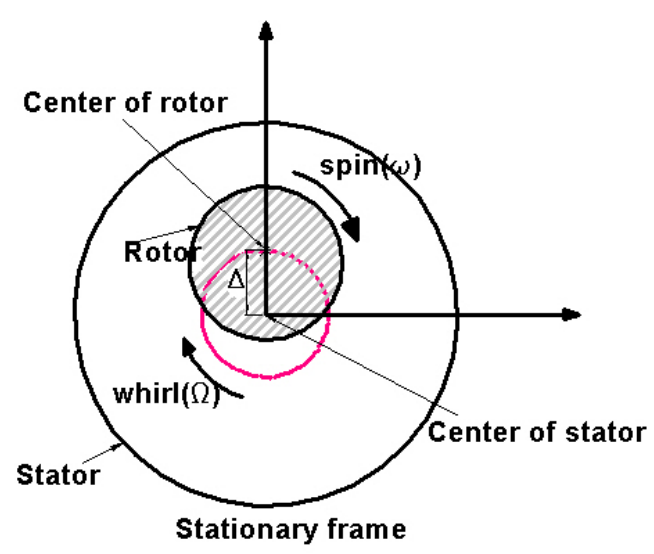

Fig.3 Stationary frame.

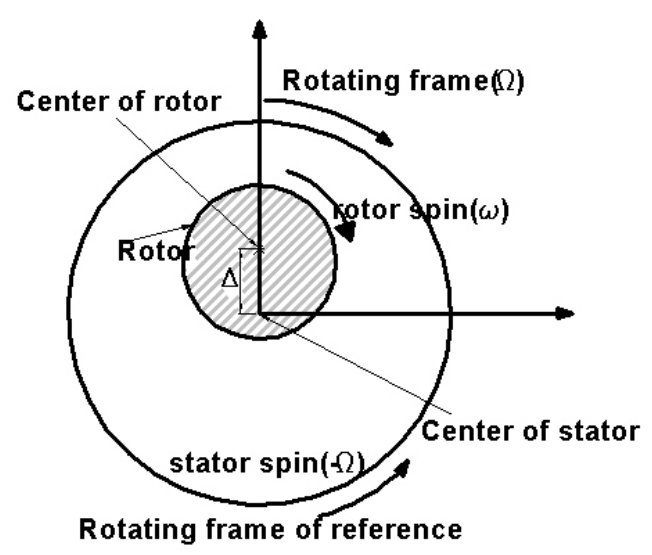

Fig.4 Rotating frame of reference.

Spinning speed of the shaft was set at $36 \mathrm{rad} / \mathrm{s}$, but the whirl speeds of the bearing were taken as 18,36 , $55,75,100 \mathrm{rad} / \mathrm{s}$. Simulations have been performed for a set of whirl ratios of $0.5,1,1.5,2$ and 2.8. The radial and tangential forces are obtained by multiplying dynamic pressure with face area in radial and tangential direction at each node respectively. Neglecting the effect of inertia, equation for forces in radial and tangential directions can be written (equations (3) and (4)). Direct stiffness $(K)$ and cross-coupled damping $(c)$ can be calculated by plotting a graph between $F_{r} / \Delta$ and $\Omega$. Similarly, cross-coupled stiffness (k) and 
direct damping $(C)$ can be calculated by plotting a graph between $F_{t} / \Delta$ and $\Omega$. One sample graph has been shown in Fig. 5. The numbers in the equation shown on the graph are the stiffness and damping coefficients.

$$
\begin{aligned}
& \frac{F_{r}}{\Delta}=-K-c \Omega+M \omega^{2} \\
& \frac{F_{t}}{\Delta}=-k-C \Omega+m \omega^{2}
\end{aligned}
$$

where, $F_{\mathrm{r}}$ is Radial force $(\mathrm{N}), F_{\mathrm{t}}$ is Tangential force $(\mathrm{N}), \Delta$ is eccentricity $(\mathrm{m}), K$ is direct stiffness coefficient $(\mathrm{N} / \mathrm{m}), k$ is cross stiffness coefficient $(\mathrm{N} / \mathrm{m}), C$ is direct damping coefficient $(\mathrm{Ns} / \mathrm{m}), c$ is cross damping coefficient $(\mathrm{Ns} / \mathrm{m}), M$ is direct mass coefficient $(\mathrm{kg}), m$ is cross mass coefficient $(\mathrm{kg}), \Omega$ is whirl speed $(\mathrm{rad} / \mathrm{s}), \omega$ is spinning speed $(\mathrm{rad} / \mathrm{s})$

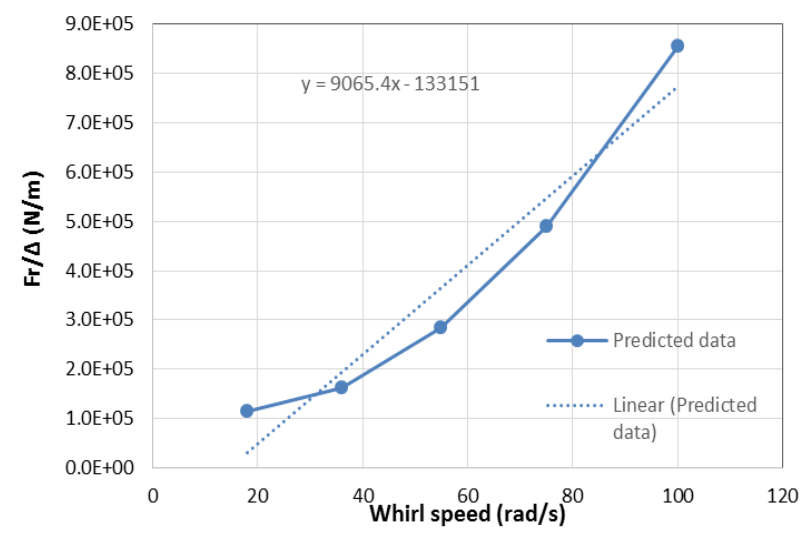

Fig.5 $F_{r} / \Delta$ versus whirl speed for spiral-grooved bearing at eccentricity ratio 0.4 .

The vibration of the rotor is described by considering the rotor to be whirling on a circular orbit about a centered position. The dynamics equation of motion for the system is written as equation (5).

$$
[M] \ddot{x}+[C] \dot{x}+[K] x=\{-F\}
$$

where, $[M]$ is mass matrix, $[C]$ is damping matrix, $[K]$ is stiffness matrix, $\{F\}$ is force vector

\section{Static and dynamic parameters for spiral- grooved bearing}

\subsection{Static pressure}

Static pressure distribution in Fig. 6 shows that both positive and negative pressures are induced in the fluid film. Maximum pressure occurs in the zone of minimum film thickness. Dynamic pressure distribution has been shown in Fig. 7. The dynamic pressures are used to calculate dynamic parameters such as the stiffness and damping coefficients.
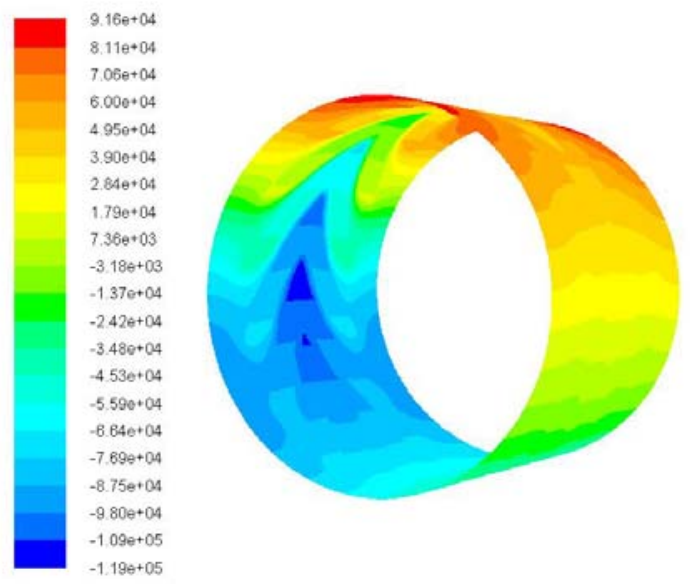

Fig.6 Static pressure contour for eccentricity ratio 0.4 .
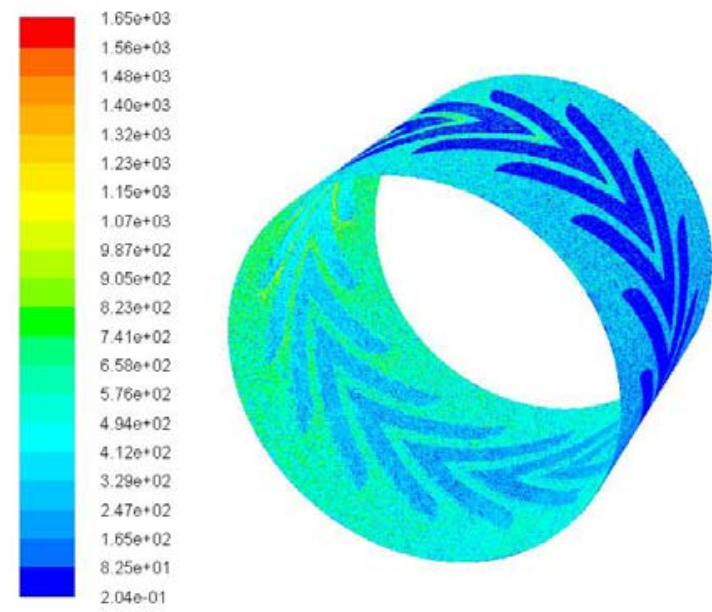

Fig.7 dynamic pressure contour for eccentricity ratio 0.4 .

\subsection{Load carrying capacity}

Like a journal bearing, the load carrying capacity of a spiral-grooved bearing increases with eccentricity ratio. Load carrying capacity is an important parameter in bearing selection. It can be calculated 
by integrating the static pressure over the rotor surface area. The load carrying capacity has been normalised by dividing it by $2 p_{a} l_{0} r_{0}$ to get dimensionless load carrying capacity.

The values of atmospheric $\left(p_{a}\right)$, length of the bearing $\left(l_{0}\right)$ and radius of the bearing $\left(r_{0}\right)$ are $101325 \mathrm{~Pa}$, $86 \mathrm{~mm}$ and $50 \mathrm{~mm}$ ([12]). In Fig. 8, the CFD predicted and the experimental values of nondimensional load for different eccentricity ratios have been presented. The experimenal data has been taken from the reasearch paper of [12]. The predicted values differ from the experimental values with a maximum error of $9 \%$.

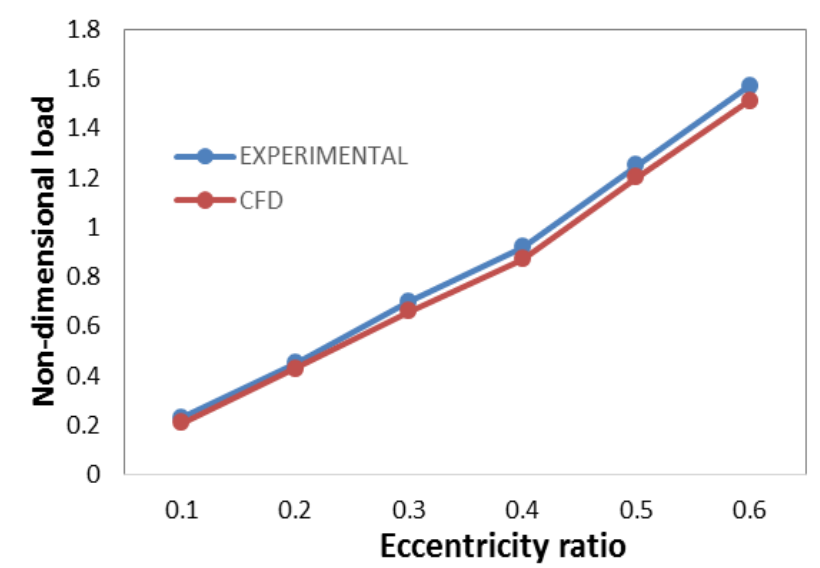

Fig.8 Experimental versus predicted nondimensional load for spiral-grooved bearing.

\subsection{Non-dimensional direct stiffness coefficient}

Stiffness coefficients have been predicted by using the dynamic pressure data calculated from the CFD simulation. The stiffness coefficient indicates the amount of resistance offered by the lubricant towards deformation due to motion of the journal. In this case, direct stiffness coefficient has been normalised by dividing it by $2 p_{a} l_{0} r_{0} / c_{r}$ to get dimensionless stiffness coefficient. Fig. 9 shows the variation of the experimental and predicted values of non-dimensional stiffness coefficients at different eccentricity ratios. It shows that the errors between the experimental and the predicted values are large for eccentricity ratios 0.2 to 0.4 .

\subsection{Damping coefficient}

Damping coefficients have been calculated using the dynamic pressures predicted from the CFD simulation. Significance of the damping coefficients is the ability of oil to reduce the oscillations of the journal. The damping coefficient reduces with the eccentricity ratio as shown in Fig. 10.

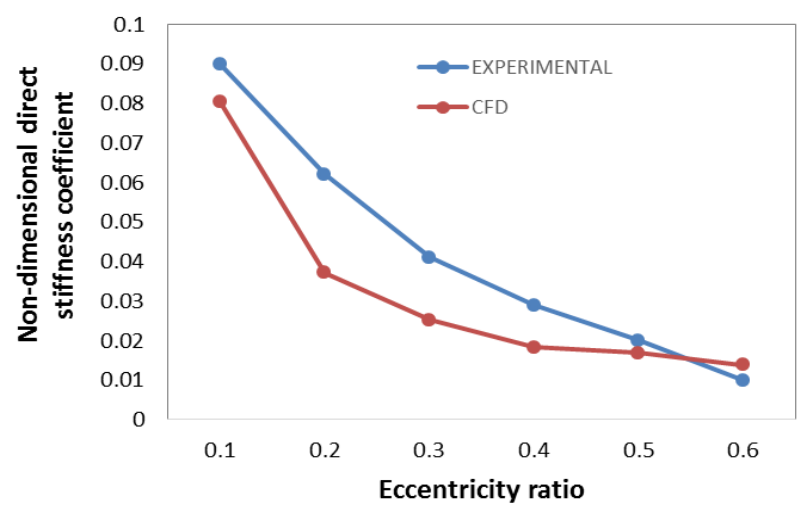

Fig.9 Experimental versus predicted nondimensional direct stiffness coefficient for spiralgrooved bearing.

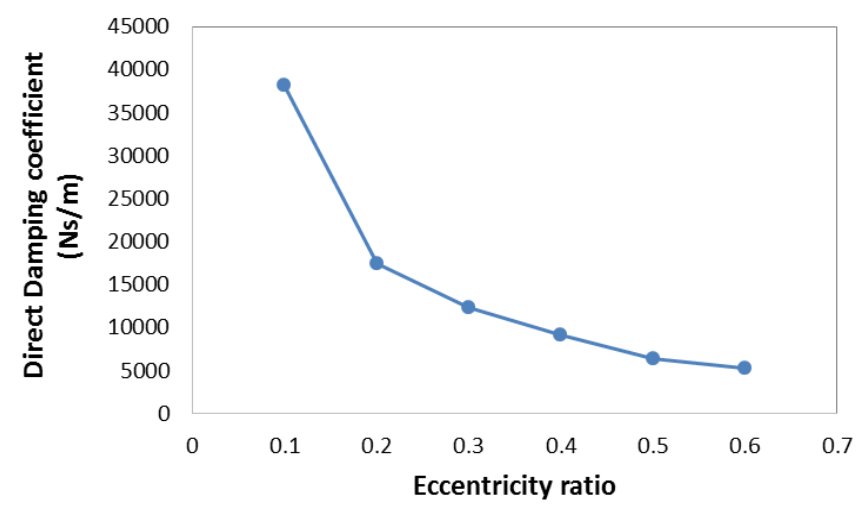

Fig.10 Predicted direct damping coefficient for different eccentricity ratios for spiral-grooved bearing.

\section{Comparative analysis of spiral-grooved bearing and plane bearing}

Fig. 11 shows the variation of a non-dimensional load with eccentricity ratio between the spiral and plain journal bearing. The spiral-grooved bearing shows lower load carrying capacity than a plain bearing. The load carrying capacity of a spiralgrooved journal bearing reduces due to higher local film thickness at grooves.

A variation of non-dimensional stiffness coefficient with eccentricity ratio for spiral-grooved bearing and plain bearing has been shown in Fig. 12. For eccentricity ratios $0.1,0.2,0.3$ and 0.4 , the difference of stiffness coefficient (between plain and spiral bearing) lies within a margin of $\pm 8 \%$. For eccentricity ratios 0.5 and 0.6 , the spiral bearings 


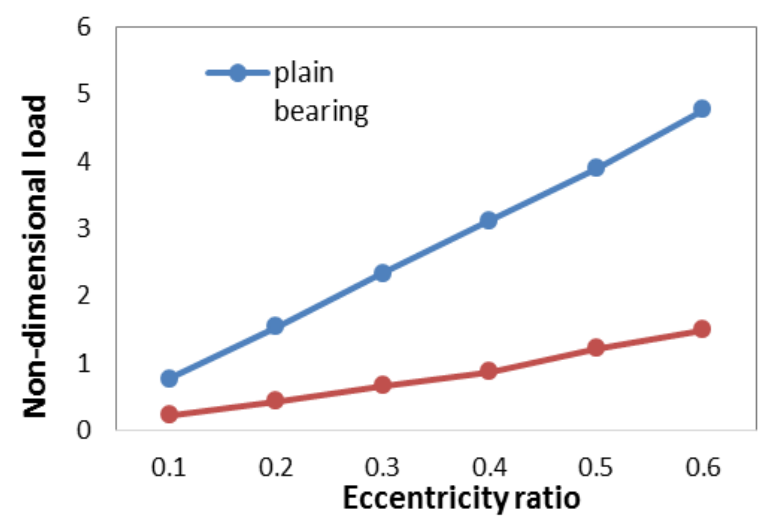

Fig. 11 Non-dimensional load compared between spiral-grooved versus plain bearing.

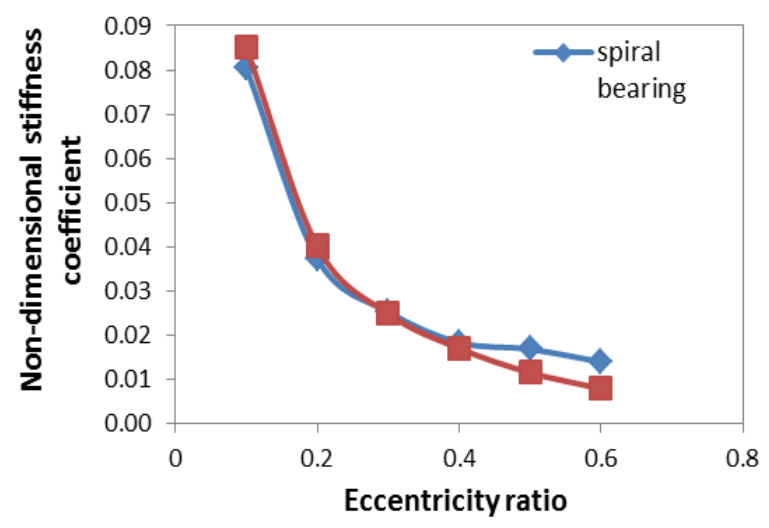

Fig. 12 Non-dimensional stiffness coefficient compared between spiral-grooved versus plain bearing.

have higher stiffness coefficient (difference is more than $30 \%$ ) than a plain bearing.

For the spiral-grooved bearing and the plain bearing, the damping coefficient variation with eccentricity ratios has been shown in Fig. 13. For all eccentricity ratios, the spiral-grooved bearing shows higher damping coefficient than a plain bearing. hence more stable.

\section{Conclusion}

In the present work the Computational Fluid dynamics (CFD) technique has been implemented to study the static and dynamic characteristics of the spiral-grooved journal bearing with grooves cut on the rotor surface. Errors between experimental and predicted values of load carrying capacity were very low. The performance of the spiral-grooved bearing and the plain bearing has been compared using the CFD technique. The spiral-grooved journal bearings

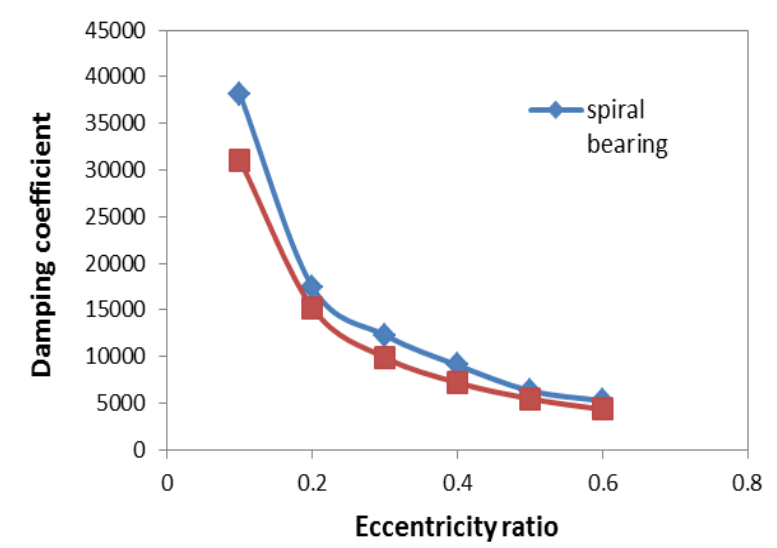

Fig. 13 Damping coefficient compared between spiral-grooved versus plain bearing.

show a lower load carrying capacity than a plain journal bearing. At eccentricity ratios 0.5 and 0.6, the spiral-grooved bearings induce a higher stiffness coefficient than plain journal bearings. But the spiral-grooved bearing shows higher damping coefficient than the plain journal bearing for eccentricity ratios 0.1 to 0.6 .

\section{References}

[1] Khan, M.Z.: Performance studies of oil lubricated helical groove journal bearing, MPhil Thesis, Brunel University School of Engineering and Design, 1992.

[2] Shelly, P., Ettles, C.: Solutions for the load capacity of journal bearings with oil grooves, holes, reliefs or chamfers in non-optimum positions, Proceedings of the Institution of Mechanical Engineers, C56 (1971), 71, pp. 3846.

[3] Vijayaraghavan, D., Keith, T.G.: Effect of type and location of Oil Groove on the Performance of Journal Bearings, Trib. Trans., 35 (1992), pp. 98-106.

[4] Roy, L., Kakoty, S.K.: Optimum groove location of hydrodynamic journal bearing using genetic algorithm, Adv. in Tribology, Article Id 580367, 2013.

[5] Ranjan, V., Pai, R., Hargreaves, D.J.: Stiffness and damping coefficients of 3-axial grooved water lubricated bearing using perturbation technique, Proceedings of fifth EDF \& LMS poitiers workshop: bearing behavior under unusual operating conditions, Futuroscope, 2006. 
[6] Pai, R., Hargreaves, D.J., Brown, R.: Modelling of fluid flow in 3-axial groove water groove bearing using computational fluid dynamics, $14^{\text {th }}$ Australian fluid mechanics conference, Adelaide University, Australia, 2001.

[7] Gertzos, K.P., Nikolakopoulos, P.G., Papadopoulos, C.A.: CFD analysis of journal bearing hydrodynamic lubrication by Bingham lubricant, Tribology International, 41(2008), 12, pp. 1190-1204.

[8] Fernandes, N., Shenoy, B.S., Pai, B.R., Pai, B.R.S., Rao, D.S.: Evaluation of stiffness and damping coefficients of multiple axial groove water lubricated bearing using computational fluid dynamics, World Academy of Sc. Eng. and Tech., 70(2012), pp. 876-881.

[9] Patil, R., Dhagat, S.K.: A Comparative CFD Analysis of a Journal Bearing with a Microgroove on the Shaft \& Journal, Proceedings of International Conference on Innovation \& Research in Technology for Sustainable Development, 2012, pp. 01-03.
[10] Dhande, D., Pande, D.W., Chatarkar, V.: Analysis of pressure for 3-lobe hydrodynamic journal bearing, Int. Jnl. of Eng. Trends and Tech., 4(2013), 8, pp. 3297-3300.

[11] Biswas, N., Pande, K.M.: Transient CFD Analysis of Multi-Lobe Bearings at 60000 RPM for A Gas Turbine, IACSIT Int. Jnl. of Eng. and Tech., 3(2011), 5, pp. 512-517.

[12] Hirayama, T., Sakurai, T., Yabe, H.: A theoretical analysis considering cavitation occurrence in oil lubricated spiral grooved journal bearings with experimental verification, Jnl. of Tribology, 126(2004), 3, pp. 490-498.

[13] Kranjcevic, L., Carija Z., Fucak S.: Numerical mesh impact on computational simulation efficiency, Eng. Review, 27(2007), 2, pp. 2536.

[14] Omar, Z.K., Mohammed, H., Kamel, H.M.: Computational aerodynamic performance of mixed-flow turbine blade design, Eng. Review, 37(2017), 2, pp. 201-213. 